\title{
La Trascendencia en Primero Sueño: el Incesto y el Aguila
}

En "Primero Sueño" hay dos aspectos que sobresalen, que trataremos de analizar: el mito del incesto y el sĭmbolo del águila. El estudio de estos aspectos tendrá como punto de partida algunos enunciados teóricos del análisis junguiano, asï como la localización del concepto "razón" en su época y cómo este concepto funciona con los símbolos ya mencionados.

En este poema Sor Juana inscribe la visión de un viaje sicológico que va desde el mundo de lo inconsciente hasta el mundo de lo que para aquella época-el siglo XVII-era lo inteligible, el mundo de la razón.

Cuando usamos la palabra, o concepto, "razón" nos enfrentamos con un problema de interpretación. Por lo general, lo primero que hacemos es interpretar este término como lo conocemos hoy día, esto es, el método deductivo separado de toda emoción y voluntad, no teniendo en cuenta que en la Europa del siglo XVII-el lente por el que miraban los intelectuales de la América Hispana de la épocaeste concepto era usado de una manera muy amplia. Los pensadores de esa época utilizaban este concepto como algo que incluía la capacidad de trascender una situación inmediata, "to grasp the whole, and such functions as intuition, insight, poetic perception were not rigidly excluded.... 'irrational' was included in their idea of reason." 1

Si vemos a "Primero Sueño" desde este ángulo podemos comprender el viaje sicológico inscrito en el poema: el deseo de llegar al conocimiento de la totalidad, la sinceridad al expresar el no poder conseguirlo $\mathrm{y}$, finalmente, la deducción de que había que dividir la realidad en las categorìas aristotélicas para poder llegar a un conocimiento de la totalidad. 


\section{El incesto como trascendencia.}

Los deseos incestuosos, de acuerdo con Jung, tanto en niños como en adultos, deben interpretarse en su contenido simbólico como una expresión del anhelo de regresar a lo primordial, al estado paradisíaco de inconciencia donde se está libre de responsabilidades y decisiones, y este lugar es el vientre de la madre. Esta tendencia regresiva no tiene solamente un aspecto negativo, sino un aspecto positivo también, en el que se implica la posibilidad de superar la atadura personal con la madre verdadera y se transfiere la energỉa síquica almacenada a este vinculo de contenido arquetípico. ${ }^{2}$ En esta etapa la libido regresiva tiende a perder su contenido de carácter sexual y expresar el problema de incesto en grandes metáforas arquetípicas de la humanidad, que se acercan a lo primordial de lo maternal y al mismo tiempo señalan el camino a la liberación de su aspecto seductivo-devorador, o sea, un 'renacimiento'. Aunque el tabú en que se ha envuel to al incesto desde tiempos inmemoriales se demuestra en la gran tentación que produce, y solamente puede ser combatido por medio de estrictas prohibiciones, no se puede negar que lo que en el plano biológico es un pecado, en el plano simbólico puede ser un acto muy significativo y hasta necesario. ${ }^{3}$

Miraremos el mito del incesto en "Primero Sueño" desde este último aspecto teniendo en cuenta la forma abierta y positiva en que se desarrolla el poema. Este va progresivamente de la oscuridad a la luz y encontramos un deseo de superación-que representa el águila y que más tarde discutiremos-que se resuelve en el poema de una manera muy peculiar, por medio de la razón, que más adelante discutiremos también. Así vemos cómo todo el transcurso del poema es positivo; por lo tanto consideramos que debe de analizarse de una manera positiva.

Sor Juana nos presenta la oscuridad envuelta de misterio,

Piramidal, funesta, de la tierra

nacida sombra, al Cielo encaminaba

de vanos obeliscos punta altiva

escalar pretendiendo las Estrellas

La tenebrosa guerra

que con negros vapores le intimaba

la pavorosa sombra fugitiva 4

De aquí pasamos a un lugar de más luz y que finalmente acaba con la 
salida del sol y el despertar. Con respecto a lo susodicho, Norman Winski, en su libro Understanding Jung, nos aclara:

Behind all myths dealing with the triumph of day over night and the battle of dark forces with the forces of light is the story of consciousness struggling to overcome the grip of unconsciousness. .. . The primary functions of consciousness is twofold: illumination and adaptation; consciousness throws light on the individual's inner needs of the moment and the opportunities and obstacles that either block or promote adapting them to outside reality. 5

Dentro de este trayecto de la oscuridad a la luz transcurre todo el proceso de trascendencia en el poema. Asï aparecen los obstáculos mayores que son los deseos incestuosos:

Con tardo vuelo y canto, del oído mal, y aun peor el ánimo admitido

la avergonzada Nictimene acecha de las sagradas puertas los resquicios, o de las claraboyas eminentes los huecos más propicios que capaz a su intento le abren brecha, y sacrilega llega a los lucientes faroles sacros de perenne llama que extingue, si no infama, en licor claro la materia crasa consumiendo, que el árbol de Minerva de su fruto, de prensas agravado congojoso sudó y rindió forzado. 6

La mitología griega nos dice que Nictimene, la hija de Nicteón, cometió incesto con su padre sin éste saber quién era ella; al saberlo trató de matarla, pero la diosa Atena la convirtió en una lechuza. Atena, esto es, Minerva en la mitologia romana, nació de la cabeza de Júpiter y es símbolo de la sabiduria ${ }^{7}$ y su árbol, el olivo, es símbolo de la paz ${ }^{8}$ y el amor divino. ${ }^{9}$ Todos estos elementos implican trascendencia, y como bien apunta Jung en Symbols in Transformation, "the effects of incest-taboo and of the attempts at canalization" de la libido, 10 "is to stimulate the creative imagination, which gradually opens up possible avenues for the self-realization of libido. In this way the libido becomes spiritualized." 11 Pudiéramos decir que en el poema se trata de trascender a la imagen del padre, o lo masculino, 
canalizando o transformando la energía que surge del conflicto en el águila. Ahora sólo tenemos una presentación del conflicto, cargado de tensión, que más tarde se resolverá en la trascendencia a través del símbolo del águila, como ya hemos dicho.

El mismo proceso del incesto lo tenemos de nuevo planteado en el poema:

El de sus mismos perros acosado,

monarca en otro tiempo esclarecido,

tîmido ya venado,

con vigilante oỉdo.

del sosegado ambiente

al menor perceptible movimiento

que los átomos muda,

la oreja alterna aguda

y el leve rumor siente

que aun lo altera dormido 12

Nos encontramos aquí con el deseo incestuoso de la hermana y el hermano, o de la madre y el hijo, de acuerdo como tomemos la historia, ya que Sor Juana le dio una forma a este mito que incluye las dos variaciones que hemos encontrado, pues nos parece difícil poderle dar la vuelta que Pfandl le ha dado. Estas variaciones nos las presenta Jung en Mysterium Coniunctionis. En primer lugar nos encontramos con la diosa Diana emergiendo de un bosquecillo y bañándose en las aguas milagrosas. Un prìncipe, Sol o Apolo, al pasar por allǐ la espía, se enamoran locamente uno del otro y ella se desmaya y cae en el agua hundiéndose; el séquito del prǐncipe rehusa salvarla por temor a lo peligroso de las aguas y entonces el principe se lanza al agua y es arrastrado a las profundidades. Inmediatamente sus almas aparecen en la superficie. 13

En la otra versión tenemos a la diosa Diana bañándose y a Acteón, el cazador, espiándola secretamente mientras ella se baña. Ella, al darse cuenta de esto, lo transforma en ciervo, a lo que los propios perros de Acteón, no reconociéndolo, lo desbaratan a pedazos. 14 Aquí tenemos el incesto de la madre y el hijo como bien apunta Pfandl15, ya que Diana es la diosa, y por ende madre de la caza y Acteón, el hijo, el cazador que es protegido y guiado por su madre espiritual. Es muy probable que Sor Juana haya conocido las dos variantes de la historia, pues no podemos explicar cómo ella utiliza gran parte del contenido de esta última versión y al mismo tiempo inserta el "monarca en otro tiempo esclarecido".16 Al conocer la existencia de estas dos variantes no podemos aceptar la 
explicación sicológica que nos brinda Pfandl ya que él usa como punto de apoyo la última versión y achaca la aparición del "monarca" al inconsciente de la poeta diciéndonos:

Una nueva variante surge del cĭrculo de representaciones y del estrato movedizo del complejo de Edipo; más ya desvanecido, por así decirlo, en su origen por una pater imago profundamente venerada, en la que la vista espiritual de la soñadora se queda un rato hechizadamente prendida. El rey perseguido y convertido en ciervo no es otro que el cazador Acteón. El tuvo el atrevimiento de sorprender en el baño a la diosa Diana, protectora y madre de todos los cazadores, y se hizo culpable, por consiguiente, de incestuosos propósitos y, en castigo de esto, es transformado en ciervo por la ofendida madre-diosa y los propios perros del cazador le persiguen sin reconocerlo y lo destrozan a dentelladas. Pero este fin trágico se avenía muy mal en la serie inconsciente de pensamientos del sueño diurno, que sólo estaba aquí dispuesto a soportar ideas seductoras; por eso será 'olvidado'. El ciervo sigue pues con vida y se convierte en el símbolo de un guardián regio, por la misma razón también que el sencillo cazador del mito quedó realzado como rey. 17

En la lïnea en que Sor Juana se refiere al "monarca en otro tiempo esclarecido", 18 bien puede haberse referido ella a la otra variante del mito, esto es, a Apolo y Diana. De esto no tenemos certeza pero consideramos que tampoco se debe pasar por alto, aunque no podremos seguir esta discusión ya que nos desvía del curso de nuestro estudio que es el tema del incesto. En el caso de Nictimene lo masculino triunfa sobre lo femenino, y en el caso de Diana, lo femenino triunfa sobre lo masculino. Ambos, Nictimene y Acteón, son transformados en animales. Aquii cabe citar a Jung, quien refiriéndose a estos mitos en el tema del incesto nos dice:

But since 'all that passes is but a parable' incest, as we have said before, is nothing but a preliminary form of the unio oppositorum. 19

Unio oppositorum, o sea unión de opuestos, en este caso lo masculino y lo femenino en la psique del individuo, o en el cuerpo del poema en este poema. Esto, de acuerdo con Jung, mantiene el equilibrio síquico, y en este caso, el equilibrio de opuestos en el poema y lo pone en el camino de la actualización, esto es, de llegar a la pleni- 
tud de ser que vemos representada en el símbolo del águila, como explicaremos más adelante. El águila, en el contexto sorjuanesco, une en una forma trascendida lo masculino y lo femenino.

II. El águila como símbolo de trascendencia y gradación de los niveles del Ser.

Una vez que salimos de la oscuridad y lo confuso en que se plantea el incesto, Sor Juana nos presenta el águila:

De Júpiter el ave generosa

-como al fin Reina-, por no darse entera

al descanso, que vicio considera

si de preciso pasa, cuidadosa

de no incurrir de omisa en el exceso,

a un solo pie librada fía el peso,

y en otro guarda el cálculo pequeño

-despertador reloj del leve sueño-,

porque, si necesario fue admitido,

no pueda dilatarse continuado,

antes interrumpido

del regio sea pastoral cuidado.

¡Oh de la Majestad pensión gravosa,

que aun el menor descuido no perdona!

Causa, quizá que hecho misteriosa,

circular, denotando, la corona,

en círculo dorado,

que el afán es no menos continuado. 20

En esta estrofa nos encontramos con un simmbolo de varios niveles de significado. Por un lado tenemos que Júpiter, Zeus en la mitologìa griega, a menudo se acercaba a la mujer que deseaba tomando la forma de águila; 21 por el otro tenemos al águila tres veces más en el poema, culminando con la imagen del "águila Evangélica" (fábrica portentosa/ que, cuanto más altiva al Cielo toca,/ sella el polvo la boca/ -de quien ser pudo imagen misteriosa/ la que Aguila Evangélica, sagrada/ visión en Patmos vio, que las Estrellas/ midió y el suelo con iguales huellas) ${ }^{22}$ seguida de otra imagen del capítulo I del Apocalipsis: 23 el hombre ceñido de oro con los pies de barro que es al alfa y el omega.

En la primera imagen del águila éste está agarrando un "cálculo pequeño", esto es, una piedra. Con respecto a la piedra nos dice von Franz en Man and his Symbols que "the stone symbolizes what is 
perhaps the simplest and deepest experience-the experience of something eternal that man can have in those moments when he feels immortal and unalterable."24 También en el capitulo II: 17 del Apocalipsis nos dice el apóstol Juan en su visión: "Arrepiéntete, pues; si no vendré a ti pronto y pelearé contra ellos con la espada de mi boca. ... al que venciere le daré del maná escondido y le daré una piedrecita blanca, y en ella escrito un nombre nuevo, que nadie conoce sino el que lo recibe." Esto parece conectar el sỉmbolo del águila con la piedra en los versos de la estrofa antes mencionada ( $\mathrm{OH}$ de la Majestad pensión gravosa,/ que aun el menor descuido no perdona! ). Finalmente en la glosa a la Virgen Marìa en el año 1683, con la que Sor Juana ganó un lugar meritorio en un certamen de poesía, ella equipara a la Virgen Marỉa con el águila:

Con luciente vuelo airoso,

Reina de las aves bellas,

fabrica entre las Estrellas

el ileso hermoso nido.

Mírala el Dragón furioso;

pero, aunque con odio intenso

mal seguirá el vuelo inmenso

del águila coronada

Mal su anhélito ha intentado

el nido infestar, que ha visto,

porque con la Piedra Cristo

quedó el nido preservado.

Qué bien el Ave burló

de sus astucias lo horrendo, pues su Concepción aun viendo, su Preservación no vio!

Bien su necedad pensó, que era el Aguila escogida de su veneno vencida, aunque miraba, en su daño, mil señales de su engaño, pero ninguna sentida. 25

Aquî tenemos sǐmbolos semejantes a los de "Primero Sueño". Por un lado el ave de Júpiter es aquí la Virgen Marìa, por el otro, la "Piedra 
Cristo" puede bien ser la piedra que tiene el águila en la pata que despierta del "leve sueño"-esto es, que trae la verdad y la justicia. Por último, ambas águilas, la de "Primero Sueño" y la de la glosa, tienen coronas. Notemos que el águila de la estrofa que estamos estudiando en "Primero Sueño" encierra el sìmbolo de la Virgen y el de Júpiter, esto es lo masculino y lo femenino trascendido. Aquï podemos decir que está la unión de símbolos opuestos de que hablamos anteriormente, pero en una forma trascendida, espiritual, en el águila que encierra a la Virgen María y a Júpiter, símbolos estos de trascendencia máxima.

Tomando la sicologĭa junguiana como medida podemos decir que en el poema se trascienden los opuestos al unirlos en el águila de Júpiter (y Marìa) y que luego comienza a fluir hacia la idea del sueño en el cuerpo del poema presentándonos el aspecto que a todos iguala: el sueño y la muerte, ya que es peculiar tanto al más alto como al más bajo desde Cristo, "quién tres forman corona"-alegoría de Cristo y que encontramos en Splendor Solis de Trismonia (1598) como apunta Jung en Mysterium Coniunctionis y nos cita el texto:

'Whoever saves me shall live and reign with me forever in my brightness on my royal throne', and night enveloped all things. The day after, they saw over the King an apparent Morning Star, and the light of Day clear up the darkness, and bright Sunlight pierce through the clouds, with manifold coloured rays of brilliant brightness, and sweet perfume from the earth, and the Sun shining clear. Herewith was completed the Time when the King of the Earth was released and renewed, well apparelled, and quite handsome, surprising with his beauty the Sun and Moon. He was crowned with three costly crowns, the one of Iron, the other of Silver, and the third of pure Gold. The saw in his right hand a Scepter with Seven Stars, all of which gave a gold Splendour. ....(etcetera)"26

hasta el ser más pobre,

pues su nivel, en todo poderoso,

gradúa por exentos

a ningunas personas,

desde a quien tres forman coronas

soberana tiara,

hasta la que pajiza vive choza 27 
De aquí pasa Sor Juana a la descripción del cuerpo humano en el sueño siguiendo las ideas del alquimista o médico Galeno y luego a la autocreación del alma que "ve en sỉ como una centella de la luz que es el Ser (Dios) hecha a imagen y semejanza de la divinidad" 28 y cree poder colocarse sobre la cumbre del monte Atlas, pero el águila no puede alcanzar apenas un tercio de la altura de este monte,

A la región primera de su altura

(infima parte, digo, dividiendo

en tres su continuado cuerpo horrendo),

el rápido no pudo, el veloz vuelo

del águila-que puntas hace al Cielo

y al Sol bebe los rayos pretendiendo

entre sus luces colocar su nido-

llegar; bien que esforzando

más que nunca el impulso, ya batiendo

las dos plumadas velas, ya peinado

con las garras el aire, ha pretendido,

tejiendo de los átomos escalas,

que su inmunidad rompan sus dos alas 29

Toma el águila dimensiones humanas que no denotan caìda sino esfuerzo, detalle muy peculiar en todo este poema, ya que lo que pudiéramos llamar derrotas son meras caídas para levantarse de nuevo. Pudiera decirse que esto es parte del proceso de llegar a la totalidad de ser, y el sol es sìmbolo de este último concepto, 30 pero al mismo tiempo teme, ya que todavía no se considera capacitada para alcanzar el Todo: la unidad o totalidad, el conocimiento de Dios.

Al sentirse impedida de llegar a la totalidad espiritual hay un quejoso regreso y una protesta de lo vano de todo esfuerzo,

Las Pirámides dos-ostentaciones

de Menfis vano y de la Arquitectura

úl timo esmero, si ya no pendones

fijos, no tremolantes-, cuya altura

coronada de bárbaros trofeos. 31

Nos habla de la ostentación vana para más tarde reconocer que las pirámides, de acuerdo con Homero, son signos exteriores de la estructura interna del alma, o, como apunta Cirlot, "la pirámide expresa la totalidad de la obra creadora, polarizada en tres aspectos esenciales."32 Interpretando esta parte Xirau nos dice: "como pirámide, 
en efecto, asciende el alma hacia la causa primera donde residen todas las esencias."33 Pero el alma puede captar esto con la intuición, volar más alto que las pirámides físicamente hablando, "Montes dos artificiales", 34 pero no puede entenderlo todo con el entendimiento y esto hace al alma retroceder. Aquí el alma y el águila son una misma cosa:

a la mental pirámide elevada

donde-sin saber cómo-colocada

el Alma se miró

En cuya casi elevación inmensa, gozosa más suspensa, suspensa pero ufana, y atónita aunque ufana, la suprema de lo sublunar Reina soberana, la vista perspicaz, libre de anteojos, de sus intelectuales bellos ojos ( $\sin$ que distancia tema ni obstáculo opaco se recele, de que in terpuesto algún objeto cele), cuyo inmenso agregado, cúmulo incomprensible, aunque a la vista quiso manifiesto dar señal de posible a la comprensión no, que-entorpecida con la sobra de objetos, y excedida de la grandeza de ellos su potenciaretrocedió cobarde. 35

El alma o águila teme continuar su experiencia hacia la totalidad, como en muchos de los recuentos de la mitología clásica y cristiana en que tenemos el temor ante la inmensidad de la Creación o el santo temor de Dios. Este temor la hace retroceder ya que no puede entenderlo todo. Debido a esto trata de usar el camino aristotélico, o sea, las diez categorias de Aristóteles (substancia, calidad, cantidad, relación, determinación espacial, determinación temporal, acción, pasión, posición y condición) ${ }^{36}$ para llegar a la comprensión de la totalidad "asi como en una escala, el alma va de lo particular a lo general, de lo concreto a lo abstracto, fiándose del intelecto metódico, claro y preciso aun dentro de los lỉmites de su finitud."37 De esta forma el entendimiento trata de entender al hombre, al mi- 
crocosmos donde se unen lo natural y lo sobrenatural, y que reune al "bruto", a "la planta" y al "ángel"-y aquí el concepto de la razón de que hablamos al comienzo de este capítulo.

Tenemos que tener en cuenta de que Sor Juana se nutre de dos colosos de la Edad Media: San Agustǐn y Santo Tomás. Con sólo leer su "Carta Atenagórica" nos podemos dar cuenta de ello. El primero le brinda las bases del racionalismo y de la filosofía moderna como ha apuntado Randall con respecto a San Agustǐn, 38 bases que sistematizó Descartes y que Sor Juana intuyó como se puede notar en la "Carta Atenagórica", en éste y otros poemas. Santo Tomás brinda a Sor Juana el sistema aristotélico cristianizado. De esta combinatoria, mutatis mutandis, sale ese racionalismo sorjuanesco que se expresa en un tratar de conocer las causas máximas a través de la razón. Veamos lo que nos dice Randall con respecto a estos dos filósofos. De San Agustín nos dice que el objeto característico del conocimento, en el sistema agustiniano, es el alma y Dios. El humano es un ser racional, y su fin natural es el de conocer la Verdad, "that sapientia or wisdom which is the Logos or Word of God himself."

The realm of Truth is thus to be approached by turning within the soul, within oneself. And so Augustine undertakes an acute and subtle analysis of this fact of knowledge as it exists within the soul. He brings remarkable psychological penetration to bear on establishing his distinctive version of the Platonic theory of knowledge. His method had momentous influence, for it was the form in which the great Platonic philosophy was transmitted to the Middle Ages and the seventeenth century.

The most certain thing about man is that he is a thinker, a rational soul using a mortal and earthly body, an animus. The highest part of this soul, and hence of man, is mens, mind, which contains both discursive reason, ratio or dianoia and intelligentia or intellectus, Nous, direct intellectual vision. 39

Santo Tomás por otro lado brinda a Sor Juana y a su edad el lado aristotélico de la moneda,

Whatever can be experienced has certain principles in terms of which human thought can find it intelligible. The forms, the causes, the principles of things are made accessible through experience and man's reflection on it. To find those principles is the object of human knowledge, to ad- 
vance from observed facts to the reasons why. And it can be done; for though knowledge must come from experience, it comes from a rational experience with an intelligible world. What experience conveys can be put into language and expressed in words and propositions and demonstrations (...) God is not to be reached through the soul alone, as the Augustinians held, but by rational inference from sense-experience. 40

De esta combinatoria surge la sintesis que es el pensamiento sorjuanesco, que no tuvo necesariamente que haber o no leïdo a Descartes, sino que en su época estaban todos los elementos para una combinatoria similar a la del racionalismo cartesiano. Así llega Sor Juana a la utilización del concepto 'razón' en su poema que culmina en las categorías aristotélicas a través de un ser "which contains both discursive reason, ratio or dianoia, and intellegentia or intellectus, Nous, direct intellectual vision". 41

Asî llegamos al "Aguila Evangélica", el apóstol San Juan que llegó a la totalidad, "sagrada/ visión en Patmos vio, que las Estrellas/ midió y el suelo con iguales huellas,/ o la estatua eminente/ que del metal mostraba más preciado/ la rica altiva frente / y en el más desechado material, flaco fundamento hacia". 42 Aquí se completa el ciclo de la totalidad, que después de varios esfuerzos Sor Juana arriba en el poema a la imagen del "Aguila Evangélica" y a la imagen del Hombre de la revelación de Juan-el alfa y omega como podemos leer en el Apocalipsis: el Cristo trascendido. De aquí en adelante comienza a relajarse la tensión y como nos dice Jacobi del sìmbolo que, según ella, es un mediador entre las cosas incompatibles de lo consciente y de lo inconsciente,

For in transcending the opposites by uniting them in itself (only to let them separate again afterwards, so that no rigidity may ensue), the symbol maintains psychic life in a constant flux and carries onward toward its destined goal. Tension and release-as an expression of the living movement of the psychic process-are enabled to alternate in a constant rhythm. 43

Y en el viaje sicológico inscrito en el poema se comienza a regresar. Sor Juana admite en el poema la confusión que sólo puede tratar de resolver por medio de la razón. De la oscuridad a la luz-la de la razón. A la conciencia y a comenzar, quizá, un nuevo viaje bajo la luz de la razón que todo lo abarca, por lo menos para los de esa época, 
que muy bien puede simbolizar el sol que surge triunfante (Llegó, en efecto, el Sol cerrando el giro. . que con luz judiciosa / de orden distributivo" $)^{4}$ y se despierta.

Lafayette College

RAFAEL CATALA

\section{NOTAS}

1. Rollo May, Ernest Angel, Henri F. Ellenberger, eds., Existence (New York: Simon and Schuster, 1958), p. 34.

2. Arquetipo: "is a tendency to form representations of a motifrepresentations that can vary a great deal in detail without losing their basic pattern. There are, for instance, many representations of the hostile brethren, but the motiff itself remains the same." C.G. Jung, M.L. von Franz, Joseph L. Henderson, et al. Man and his Symbols (New York: Dell Publishing Co., Inc., 1972), p. 58.

3. Jolande Jacobi, Complex/Archetype/Symbol in the Psychology of C.G. Jung, Bollingen Series LVII (New Jersey: Princeton University Press, 1959), pp. 90-91.

4. Sor Juana Inés de la Cruz, Obras completas, prólogo de Francisco Monterde (México: Editorial Porrúa, S.A., 1972), p. 183.

5. Norman Winski, Understanding Jung (Los Angeles, California: Sherbourne, Inc., 1971) pp. 36-37.

6. de la Cruz, Obras completas, pp. 183-184.

7. G. A. Gaskell, Dictionary of All Scriptures and Myths (New York: The Julian Press, Inc., 1969) p. 81.

8. Juan Eduardo Cirlot, Diccionario de símbolos (Barcelona, España: Editorial Labor, S.A., 1969) p. 352.

9. Gaskell, pp. 548-49.

10. En la sicologia junguiana la libido no significa energia sexual solamente, sino energìa sĭquica en general.

11. Carl G. Jung, The Collected Works of C.G. Jung, trans. R.F.C. Hull (New York: Pantheon Books, 1963), V, 224.

12. de la Cruz, Obras completas, p. 185.

13. Carl G. Jung, The Collected Works of C.G. Jung, trans. R.F.C. Hull (New York: Pantheon Books, 1963), XIV, 121.

14. Además, añade Jung en una nota al calce que puede indicarnos el trasfondo sicológico del mito: "Since a psychic transformation is involved, the obscure passase in the Naassene hymn (Hyppolytus, Elenchos, V, 10, 2), describing the sufferings of the soul, might be relevant here: 'the soul. . . veiled in the form of a stag wearies, overpowered by the pains of death.' *But the text is so uncertain that it has little documentary value." Jung, Collected Works, XIV, 159-160.

15. Ludwig Pfandl, Sor Juana Inés de la Cruz - La décima musa de México: su vida, su poesía, su psique, ed. Francisco de la Masa (México: Instituto de Investigaciones de México, 1963), p. 215. 
16. de la Cruz, Obras completas, p. 185.

17. Pfandl, p. 215.

18. de la Cruz, p. 185.

19. Jung, Collected Works, XIV, 160.

20. de la Cruz, pp. 185-86.

21. Jung, Man and his Symbols, p. 265.

22. de la Cruz, p. 196.

23. Como apunta Pfandl en su libro, p. 226.

24. Jung, Man and his Symbols, p. 224.

25. de la Cruz, p. 130.

26. Jung, Collected Works, XIV, pp. 331-32, el subrayado es nuestro.

27. de la Cruz, p. 186.

28. Ramón Xirau, Genio y figura de Sor Juana Inés de la Cruz (Buenos Aires, Argentina: Editorial Universitaria de Buenos Aires, 1970) p. 147.

29. de la Cruz, p. 189.

30. Jung, Man and his Symbols, pp. 266 and 285.

31. de la Cruz, p. 189.

32. Cirlot, Diccionario de simbolos, p. 377.

33. Xirau, p. 148.

34. de la Cruz, p. 191.

35. de la Cruz, pp. 191-92.

36. Sterling P. Lamprecht, Our Philosophical Tradition (New York: Appleton Century-Crofts, Inc., 1955), p. 158.

37. Xirau, p. 151.

38. John Herman Randall, Jr. The Career of Philosophy: from the Middle Ages to the Enlightenment (New York: Columbia University Press, 1962), I, 158.

39. Randall, p. 25.

40. Randall, p. 32-33.

41. Randall, p. 25.

42. de la Cruz, p. 196.

43. Jacobi, p. 98.

44. de la Cruz, p. 201. 